\title{
Insights from Exemplar Practices on Achieving Organizational Structures in Primary Care
}

\author{
Greta Tubbesing, BS, and Frederick M. Chen, MD, MPH
}

Purpose: Interprofessional practice (IPP) is associated with better patient care outcomes and patient and provider satisfaction, yet little is known about the organizational structures that support effective IPP.

Methods: We selected 9 diverse clinical practice sites with exemplary IPP and conducted site visits with nonparticipant observations and interviewed 80 physicians, nurses, pharmacists, dieticians, medical and hospital assistants, nurse practitioners, physician assistants, clinic managers, physical and occupational therapists, respiratory therapists, social workers, psychologists, and others. We independently coded field notes and interviews and identified themes and trends using a grounded theory approach. Sites were evaluated for IPP using key features identified by the 2011 Interprofessional Education Collaboration Expert Panel.

Results: The primary themes at sites with high IPP were coordination of care and mutual respect. Four key organizational features were associated with these 2 themes: independent responsibilities for each professional; organizational structures for providers to learn about each other's roles; a structure and culture promoting accessible, frequent communication about patients; and strong leadership in IPP-supportive values.

Conclusions: To achieve interprofessional collaboration, practice teams require structural supports that facilitate coordination of care and mutual respect. (J Am Board Fam Med 2015;28:190-194.)

Keywords: Delivery of Health Care, Health Personnel, Interprofessional Care, Practice Management

The need for effective interprofessional practice (IPP) is well established. The Joint Commission cites communication failures as the leading root cause for medication errors and delays in treatment. ${ }^{1}$ IPP is associated with better access and improved patient care and safety, cost-effectiveness, and patient and provider satisfaction. ${ }^{2-4}$ The Institute of Medicine has called for collaborative interdisciplinary teams as a key aspect of health care system improvement. ${ }^{5}$ The World Health Organization (WHO) released in 2010 a call for action to move toward embedding interprofessional educa-

\footnotetext{
This article was externally peer reviewed.

Submitted 5 April 2014; revised 5 November 2014; accepted 12 November 2014.

From the Department of Family Medicine, University of Washington School of Medicine, Seattle.

Funding: This work was supported in part by the Washington Academy of Family Physicians Foundation.

Conflict of interest: none declared.

Corresponding author: Frederick Chen, MD, MPH, Department of Family Medicine, University of Washington School of Medicine, 4225 Roosevelt Avenue NE, Suite 300, Seattle, WA 98195-4982 (E-mail: fchen@u.washington. edu).
}

tion and practice in all health services. ${ }^{4}$ Most recently, the Family Medicine for America's Health project has reemphasized the patient-centered team approach and family physicians' relationships to other professionals. ${ }^{6}$

Much work has been done to identify the interpersonal and human factors present in effective IPP. In 2011 the Interprofessional Education Collaborative Expert Panel identified the knowledge, skills, and attitudes that make up the core competencies required for interprofessional practice ${ }^{7}$ : effective communication, information sharing, clarity of roles and responsibilities, and shared values. The National Interprofessional Competency Framework, developed by the Canadian Interprofessional Health Collaborative in 2010, listed 6 competency domains, which in addition to the above also include patient/family/community-centered care, collaborative leadership, and interprofessional conflict resolution. ${ }^{8}$

The WHO Framework lists the following institutional factors that enable effective IPP: institutional supports, including clear governance models, 
structured protocols, and management in support of teamwork; a working culture, including shared decision making and routine team meetings, structured information systems, and effective communication strategies; and, finally, environment, including space design that does not reflect a hierarchy and facilitates communication and collaboration. ${ }^{4}$

Previous studies looked at organizational structures of IPP in specific or isolated clinical contexts. ${ }^{9}$ Others have explored the effect of IPP on individual provider participation ${ }^{10}$ or on specific providerprovider interactions. ${ }^{11}$ No studies have examined organizational factors as they relate to IPP across practice types and locations. We sought to identify the common structural features of IPP across multiple clinical settings within our institution.

\section{Methods}

Based on a literature review of IPP, we created an observational tool and interview template. The observational tool was a checklist of known elements of effective IPP based on the literature search. The study instruments were piloted at a clinical site that was not affiliated with the University of Washington.

Ten expert key informants from medicine, nursing, and pharmacy identified clinical sites at the University of Washington Medical Center and affiliated Harborview Medical Center. ${ }^{12}$ The key informants had expert knowledge of the principles of IPP and held positions that allowed them to observe practice sites throughout the institution. These key informants were asked to identify "best practice" models of interprofessional collaboration and teamwork within the University of Washington system.

Nine sites were selected based on the number of recommendations and the diversity of practice settings. The sites included 2 general outpatient clinics, 3 specialty outpatient clinics, 2 inpatient units, an emergency department, and 1 inpatient consult team. A clinical leader at each site was contacted by E-mail and invited to participate in the study. All sites contacted agreed to participate.

We observed clinical practice at each site using the assessment tool for a minimum of 2 hours over 1 to 3 different days or shifts. During the site visits, the investigator (GT) made efforts to keep observational notes objective, without including personal experience. Organizational features noted included practice elements such as clinic layout and workstation assignments, designation of roles and responsibilities, leadership structures, trainings, and formal and informal structures for communication. Interpersonal interactions, including tone and content, were logged for later coding and analysis.

We conducted 80 interviews with team members from 12 professions, including physicians, nurses, pharmacists, dieticians, medical and hospital assistants, nurse practitioners, physician assistants, clinic managers, physical and occupational therapists, respiratory therapists, social workers, psychologists, and others. Interviews were openended. Participants were asked to describe IPP at their practice site, to identify the supporting structures at the site, and to describe what they considered the most important structural elements required to facilitate IPP. Interviews lasted between 20 to 60 minutes each and were conducted in private, with an assurance of anonymity to encourage full disclosure. Interview responses were typed in shorthand and then translated back to full syntax for analysis.

We evaluated the sites for IPP using the key features identified by the 2011 Interprofessional Education Collaboration Expert Panel: effective communication, information sharing, clarity of roles, and shared values. We used grounded theory to identify structural and organizational factors present at clinical sites that correlated with highlevel IPP. ${ }^{13-16}$ Analysis occurred throughout the process of data collection to identify emerging themes and modify the study as needed. Themes were expanded and refined as new information emerged. Selected quotations exemplified each of the themes and illustrate the themes at a personal level. The study was reviewed as exempt by the University of Washington Institutional Review Board (reference no. 43351).

\section{Results}

We identified IPP using a framework of the key features identified by the 2011 Interprofessional Education Collaboration Expert Panel: effective communication, information sharing, clarity of roles, and shared values. ${ }^{7}$ The primary themes that emerged in these high-IPP sites were coordination of care and mutual respect. The clinical teams were organized and performed in a way that prioritized care coordination between and among team mem- 
bers. At the personal level, these clinical teams all valued and demonstrated mutual respect for fellow team members, among all professions. While coordination of care and mutual respect are commonly described in IPP, we identified 4 key organizational features that enabled these exemplary IPP teams.

\section{Unique Responsibilities of Each Team Member}

IPP sites demonstrated unique and independent responsibilities for each professional, though they did not necessarily require unique knowledge or skills. It was important that individuals felt ownership of their roles, that they had some autonomy in their work, and that their responsibilities contributed to the team and to high-quality patient care. The team members described feeling self-directed, accountable, and that they had an important contribution to make toward patients' outcomes. Other team members, including those in leadership roles and with more decision-making authority, touted the contributions of all team members as essential.

"These patients are complicated, and we all have a different area of expertise. We all do what we're good at." - Social worker

"Everybody would say that they have an identity in their role." - Manager

The responsibilities did not necessarily require unique skills or knowledge. Some professionals may not have had more knowledge than physicians about their area of expertise, but they had responsibility for in-depth conversation with patients about these topics and were accepted in this role. For example, medical assistants who managed patients' immunization schedules felt this responsibility was their domain. In interviews, team members in various roles endorsed pride in their unique responsibilities, and colleagues endorsed dependence on their colleagues for their skills in these areas.

Not all independent roles involved direct patient care, but pride in individual contributions to the shared goal of high-quality care was a recurrent theme. Some clinic coordinators had very little direct involvement with patients but still took pride in their contributions to high-level care. When pharmacists were on site, physicians and others frequently consulted them, even when they did not have appointments with patients.

\section{Structure to Learn Each Other's Roles}

Each site had an organizational structure for providers to learn the nature of each other's roles. These structures took many forms and ranged from formal training to clinical rounding that mandated active participation by all team members. Some sites used formal training such as Team Strategies and Tools to Enhance Performance and Patient Safety (TeamSTEPPS), a program developed by the Department of Defense in collaboration with the Agency for Healthcare Research and Quality. ${ }^{17}$ TeamSTEPPS teaches specific language and tools that are aimed at increasing the effectiveness of team members with regard to leadership, communication, situation monitoring, and mutual support. TeamSTEPPS enforces predetermined language to create a safe, nonthreatening space for any team member to speak openly about concerns.

"[As a result of the training] people feel more engaged in their work teams. They feel more responsive and responsible to their colleagues. I think it is heightened the awareness about how each of us affects our colleagues and the whole unit." - Nurse

"Everyone has a really good idea of what everyone else does. In fact, we just had a staff meeting on Monday there were like 20 people there, and everyone said what their job title is and what they actually do. You could see the light bulbs go on: 'Oh yeah I can go to them for that.' Then they also know how what they do impacts the other people, how they impact other people's work." —Care coordinator

Other sites used a structured morning brief or "huddle" to ensure all team members have the information necessary for the day and sometimes to share learning points or points of reflection on care or mission. Sometimes these meetings did not just leave open the option of contributions but had an expectation of full presence and vocal contributions by each team member present.

"Rounds create the atmosphere where communication is easier for the rest of the day. Because everyone is involved at first and everyone has a role to play." - Physician fellow

\section{Culture of Communication}

The sites had a structure and culture of accessible, frequent communication about patients. Various forms of communication were used, including notes sent through the electronic health record, phone calls, and casual and structured in-person 
conversations. This communication often occurred in shared interprofessional workrooms or open work spaces that facilitated frequent interaction. When this was not present, a culture of frequent personal or electronic communication was especially important.

Other examples included impromptu teaching such as a psychologist's consultation for managing challenging patients or a social worker's knowledge of available resources; joint problem-solving, and an open culture of welcomed input and questions. This often facilitated personal conversation and staff getting to know each other on a more casual level, facilitating camaraderie and empathy.

"There's rarely a day when I do not talk to everyone in this clinic." - Attending physician

"We go to each other proactively." - Pharmacist

"The interprofessional model has always been more successful. It is not just medical updates, but what is driving the plan, what is happening with the plan now. We troubleshoot, we problem solve: 'This is the barrier we're running into, what would you recommend?' I propose different ideas or different language to use with the patient. There are individual areas of expertise that each provider brings, but also cross-training and carryover across disciplines in terms of what we're working on." -Vocational counselor

"The provider work area, which is shared, is where the providers 'hang out,' do their work, hear other people speaking, get shoulder-tapped, ask[ed] if they can comment on a case they have seen or ask a question. This is the space we share physically and also in our professional interactions." —Attending physician

\section{Physician Leadership}

There was strong leadership, specifically from attending physicians, who valued the contribution of each team member and communicated this either overtly or implicitly. Many of the team members described the supportive tone set by physician leaders. They created a culture within the care setting that emphasized IPP values, including patient- and family-centered care, high-quality care, a safe space for all team members to contribute, and an expectation that every team member contribute.

"You have to really respect and believe that everyone on the team has something to offer. Then
I need to be always striving to invite everyone to be heard." -Attending physician

"It is the attendings who have laid this foundation of 'This is the way we are,' and they teach our residents to be like that. They really set a good example." - Medical assistant

"The best physician leaders I've seen recognize they do not know everything and cannot do everything, and that there are experts in different kind of work. Then they acknowledge those people individually: 'I know who you are and I know how you can help.' The doctor knows the RD [registered dietitian], RT [respiratory therapist], bedside pharmacist, and says 'This is what you are good at, so I am going to use you.' Then they give feedback to everyone about the quality of their work." — Pharmacist

\section{Discussion}

We conducted a qualitative study of exemplary IPP sites at the University of Washington. Using a theoretical framework of the Interprofessional Education Collaborative Expert Panel competencies, ${ }^{7}$ our findings confirmed the importance of effective communication, information sharing, clarity of roles, and shared values in IPP. The team members in these sites had unique, independent responsibilities that contributed to a common goal of patient care. Second, organizational structures such as training or rounding enabled providers to learn about each other's roles. Third, there was frequent communication about patients. Finally, physician leaders fostered a culture of IPP.

These findings reinforced the WHO institutional supports of IPP. ${ }^{4} \mathrm{We}$ also were able to identify strategies and tools at the clinical practice level that helped to foster the culture of IPP. These included routine team meetings such as rounds and multiple modalities for frequent communication. These organizational structures and characteristics can be introduced into other care settings to foster IPP.

Certain factors were not critical to successful IPP. Interestingly, physical space was not a major factor in our study, although it is one of the WHO institutional supports. For example, physical proximity was not always necessary for effective communication to occur. In our study in-person communication could be substituted with electronic messages. 
Successful IPP does not require interpersonal friendships. Even many sites with good IPP had some degree of interpersonal conflict. This finding reinforces the importance of professional role clarity, training to understand team members' roles, and frequent communication. Although a highfunctioning team often engenders friendships, the professional nature of the work demands role clarity regardless of personal relationships. IPP is much more than the random coming together of multiple professions in a common space. In fact, IPP is characterized by the working relationships between professions and the structures that support it.

We were surprised to find the emphasis on physician leadership because the interprofessional movement has emphasized equal standing among professions. Nevertheless, it is understandable that within the historic hierarchy of medical care, physicians continue to provide clinical leadership and often are seen as the tone-setting professional in many clinical teams. We do not think this finding detracts from the shared responsibilities of all professions in the health care team.

This study has several limitations. Our data come from a single institution, although we surveyed diverse clinical sites including inpatient, outpatient, primary care, and specialty settings. Although our institution has a strong interest in interprofessional education, this was the first systematic effort to identify IPP sites and study them. As with all qualitative research, there is the potential for personal bias by investigators. The primary observer (GT) was a second year medical student at the time of the study. Although she is a physician in training, her observations took place before her immersion in clinical training, so her perspective was relatively untainted by medical professional culture.

These findings suggest practical innovations that health systems, hospitals, and clinical practices can undertake to foster better IPP. Within academic settings, these structural features may help guide clinical placements that enable students to experience and learn in high-functioning interprofessional teams.

\section{References}

1. Joint Commission on Accreditation of Healthcare Organizations. The Joint Commission guide to improving staff communication. Oakbrook Terrace (IL): Joint Commission Resources; 2005.

2. Center for Health Sciences Interprofessional Education, Research and Practice. IPE handout. Available from: http://collaborate.uw.edu/sites/default/files/ files/WSNA_Zierler.pdf. Accessed October 26, 2014.

3. Suter E, Deutschlander S, Mickelson G, et al. Can interprofessional collaboration provide health human resources solutions? A knowledge synthesis. J Interprof Care 2012;26:261-8.

4. World Health Organization, Department of Human Resources for Health. Framework for action on interprofessional education \& collaborative practice. Geneva, Switzerland: World Health Organization; 2010.

5. Institute of Medicine. Crossing the quality chasm: a new health system for the 21 st century. Washington, DC: National Academies Press; 2001.

6. Family Medicine for America's Health: future of family medicine 2.0. Available from: http://www.aafp.org/ about/initiatives/future-family-medicine.html. Accessed January 13, 2015.

7. Interprofessional Education Collaborative Expert Panel. Core competencies for interprofessional collaborative practice: report of an expert panel. Washington, DC: Interprofessional Education Collaborative; 2011.

8. Canadian Interprofessional Health Collaborative. A national interprofessional competency framework. Vancouver, BC, Canada: University of British Columbia; 2010.

9. Campbell S, Stowe K, Ozanne EM. Interprofessional practice and decision support: an organizational framework applied to a mental health setting. J Interprof Care 2011;25:423-7.

10. Pfaff KA, Baxter PE, Ploeg J, Jack SM. An integrative review of the factors influencing new graduate nurse engagement in interprofessional collaboration. J Adv Nurs 2013;70:4-20.

11. Van C, Costa D, Mitchell B, Abbott P, Krass I. Development and validation of the GP frequency of interprofessional collaboration instrument (FICIGP) in primary care. J Interprof Care 2012;26:297304.

12. Marshall MN. The key informant technique. Fam Pract 1996;13:92-7.

13. Burnard P. A method of analysing interview transcripts in qualitative research. Wales, UK: University of Wales College of Medicine; 1991.

14. Tobin GA, Begley CM. Methodological rigour within a qualitative framework. J Adv Nurs 2004;48: 388-96.

15. Glaser B, Strauss AL. The discovery of grounded theory: strategies for qualitative research. Chicago: Aldine; 1967.

16. Strauss A, Corbin J. Basics of qualitative research: grounded theory procedures and techniques. Newbury Park: Sage; 1990.

17. Turner P. Implementation of TeamSTEPPS in the emergency department. Crit Care Nurs Q 2012;35: 208-12. 